www.nature.com/ejhg

\title{
Pathogenic mutations and rare variants of the $A P C$ gene identified in 75 Belgian patients with familial adenomatous polyposis by fluorescent enzymatic mutation detection (EMD)
}

\author{
Geneviève Michils ${ }^{1}$, Sabine Tejpar ${ }^{1,2}$, Jean-Pierre Fryns ${ }^{1}$, Eric Legius ${ }^{1}$, Eric Van Cutsem ${ }^{2}$, \\ Jean-Jacques Cassiman ${ }^{1}$ and Gert Matthijs*,1
}

${ }^{1}$ Center for Human Genetics, University of Leuven, Leuven, Belgium; ${ }^{2}$ Department of Gastro-enterology, University of Leuven, Leuven, Belgium

Familial adenomatous polyposis (FAP) is a dominant inherited colorectal cancer syndrome which is caused by germline mutations in the adenomatous polyposis coli (APC) gene. Enzymatic mutation detection (EMD) has potential advantages over the standard protein truncation test (PTT) that is currently used in screening the $A P C$ gene for mutations. First we wanted to validate the EMD technique in comparison to PTT. Secondly, we wanted to develop an efficient working protocol for EMD screening of $A P C$. Seventy-five unrelated patients were screened for mutations. All mutations that had previously been detected by PTT were also identified by EMD; the sizes of the cleavage fragments were as expected according to the position of the mutations within the amplicons. A new screening strategy based on EMD allows the analysis of the APC gene in 31 overlapping PCR fragments. In total, EMD efficiently detected the 26 truncating mutations in this series. In addition, two rare variants were also detected: the first is the typical Ashkenazi missense mutation I1307K while the second variant, E1317Q, has been identifed in Belgian patients and controls, and should no longer be considered as a pathogenic mutation, but rather classified as a polymorphism.

European Journal of Human Genetics (2002) 10, 505 -510. doi:10.1038/sj.ejhg.5200825

Keywords: enzymatic mutation detection; EMD; variants; hypermutable; FAP; adenomatous polyposis coli (APC); protein truncation test

\section{Introduction}

Familial adenomatous polyposis (FAP) is an autosomal dominant disorder characterised by the development of at least 100 colorectal adenomas at a young age (before 20 years), some of which will progress to colorectal cancer one or two decades later. In addition to the classical form of FAP, an attenuated form was described: attenuated FAP (AFAP) or attenuated adenomatous polyposis coli (AAPC), in which the number of polyps ranges between 10 and

*Correspondence: G Matthijs, Center for Human Genetics, UZ Gasthuisberg, Herestraat 49, B-3000 Leuven, Belgium. Tel: +32 16346070;

Fax: +32 16346060; E-mail: gert.matthijs@med.kuleuven.ac.be Received 23 July 2001; revised 18 April 2002; accepted 23 April 2002
99, appearing at a later age and being still associated with an increased risk of cancer. ${ }^{1}$ The profuse phenotype is associated with the appearance of more than five thousand polyps, whereas patients with the classical sparse phenotype eventually develop one to two thousand polyps. The adenomatous polyposis coli (APC) gene was discovered in 1991, ${ }^{2,3}$ and the germline mutation spectrum is generally spread over its entire coding region. Hot spot regions for somatic mutations in colorectal tumors (mutation cluster region (MCR) in exon 15), ${ }^{4}$ and in desmoids (middle part of exon $15)^{5}$ are well characterised. The positions of the germline mutations correlate with the severity of the disease. For example, in classical FAP, mutations are located between 
codons $213-1249$, whereas in the profuse phenotype mutations are located between codons $1250-1464 .^{6}$ Mutations in exon 9 and at the $5^{\prime}$ and the $3^{\prime}$ end of $A P C$ are also responsible for AFAP. ${ }^{7-9}$ Known germline and somatic mutations can be found at the internet site address: http://perso.curie.fr/Thierry.Soussi/APC.html\#Ancrage3.

Due to the extended mutation spectrum of the APC gene, and the size of the gene itself, there is a need for an efficient and sensitive mutation detection method. Currently the protein truncation test (PTT) is the technique most widely used to screen for mutations in the APC gene. ${ }^{10,11}$ However, PTT only allows the detection of truncating mutations. While over $90 \%$ of pathogenic mutations detected in classic FAP are truncating mutations, missense mutations, polymorphisms and variants of unknown significance that have been reported are spread over the whole gene. The majority of these would not have been detected by PTT (e.g. E1317Q, S1971R, A2119V). ${ }^{12,13}$

We wanted to evaluate the feasibility and efficiency of enzymatic mutation detection (EMD) screening of the $A P C$ gene. This is potentially an easy, rapid and accurate method that has the advantage of detecting all types of mutations. ${ }^{14}$ EMD is based on the properties of the T4 endonuclease VII that has the ability to detect mismatches in a double-stranded DNA molecule and to cleave the DNA strand at this locus. ${ }^{15}$ The cleavage products can easily be detected after electrophoresis. EMD has been originally developed from the enzymatic mismatch cleavage method (EMC), ${ }^{16}$ and has been improved to allow extended screening.

In this study, we aimed to validate EMD by comparing it to PTT. In addition, we established an efficacious screening protocol which allows rapid scanning of all exons of $A P C$.

\section{Subjects and methods}

DNA from clinically affected FAP or AFAP patients submitted for molecular diagnostic analysis was used for this study. Lymphocyte DNA from patients and controls was prepared using standard salt precipitations.

For EMD analysis of exon 15, amplicons of different sizes were generated using several combinations of described primers. ${ }^{2}$ In addition, a few new primers were designed: c3 (forward) 5'-CACAAGCAAAGTCTCTATGGTG, h2 (reverse) 5'-TTTCTGCCTCTTTCTCTTGG, i3 (forward) 5'-ACCAAGAGAAAGAGGCAGAA. The amplicons were: a-c, c3-d, d-e, ff, g-h, h-h2, i3-i, j-k, k-l, m-n, o-p, q-r, s-t, u-v, and v-w. The remaining 16 segments of $A P C(1-9,10,11-14$, and the alternative spliced exons $9 \mathrm{~A}$ and 10A) were amplified as described. ${ }^{2,17}$ For each amplicon, both forward and reverse primers were labelled with fluorescein isothiocyanate (FITC). Fluorescently labelled PCR fragments were submitted to the EMD reaction with the Passport kit for ABI 377 detection (Amersham Pharmacia Biotech). Denaturation $\left(95^{\circ} \mathrm{C}, 4 \mathrm{~min}\right)$ was followed by a cooling step from $95^{\circ} \mathrm{C}$ to $37^{\circ} \mathrm{C}$ at $0.02^{\circ} \mathrm{C} / \mathrm{s}$. Enzyme was then added to the samples and incubation for $30 \mathrm{~min}$ at $37^{\circ} \mathrm{C}$ followed. All the incubation steps were performed in a standard thermocycler. EMD products were loaded on a 6\% Long Ranger Urea gel (SanverTech) and detected with an ALF DNA sequencer (Amersham Pharmacia Biotech). PTT analysis of exon 15 has been performed as described. ${ }^{11}$

Exon 6 has been screened for all patients using Denaturing Gradient Gel Electrophoresis (DGGE) according to Olschwang et al. ${ }^{18}$

\section{Results}

PTT was initially used for the APC exon 15 screening of a series of 40 patients. It has been performed in four overlapping fragments. In fifteen out of the 40 patients, a truncated peptide was detected; sequencing revealed ten different alterations that were all localized in the first half of exon 15.

For comparisons, these samples were submitted to EMD in amplicons of several sizes, ranging from 277 to $910 \mathrm{bp}$. The size of the cleavage peaks could be calculated according to the position of the mutation within the tested amplicon. As both primers were labelled, two peaks were expected for detection after cleavage. All peaks were positively detected and some mutations were observed in overlapping amplicons (Table 1).

The samples showing a negative PTT result have been further analysed by EMD of the exons 1-14. A second series of 35 patients, which had not been previously tested

Table 1 Germline mutations of the APC gene identified in Belgian FAP patients

\begin{tabular}{|c|c|c|c|c|}
\hline Mutation & Codon & $\begin{array}{c}\text { Exon } \\
\text { (fragment) }\end{array}$ & $\begin{array}{l}\text { Detection } \\
\text { method }\end{array}$ & Patient \\
\hline R216X & 216 & 6 & DGGE & 187832 \\
\hline $\mathrm{R} 232 \mathrm{X}$ & 232 & 6 & DGGE & 191930 \\
\hline R302X & 302 & 8 & EMD & 186794 \\
\hline 1087 ins $A$ & 357 & 9 & EMD & 202032 \\
\hline S457X & 457 & 10 & EMD & 179129 \\
\hline 1494 del C & 491 & 11 & EMD & 207230 \\
\hline R499X & 499 & 11 & EMD & $151913 ; 178620$ \\
\hline $2128 \mathrm{del}$ G & 704 & $15-a c$ & EMD & 110175 \\
\hline Y935X & 935 & $15-a c / c 3 d$ & PTT-EMD & 38378 \\
\hline 2911 del AATA & 966 & $15-c 3 d / d e$ & PTT-EMD & 30913 \\
\hline 3104 del TT & 1028 & $15-c 3 d / d e$ & PTT-EMD & 175491 \\
\hline 3201 del ACAAA & 1061 & $15-\mathrm{de}$ & EMD & 153316 \\
\hline Q1175X & 1175 & 15 -ff & EMD & 228789 \\
\hline S1194X & 1194 & $15-\mathrm{ff}$ & PTT-EMD & 180798 \\
\hline 3614 ins $A$ & 1199 & 15 -ff & PTT-EMD & 35963 \\
\hline 3945 del AAAGA & 1309 & $15-g h$ & PTT-EMD & $\begin{array}{l}\text { 167202; 167877; } \\
163927 ; 185236\end{array}$ \\
\hline $4057 \mathrm{GC} \rightarrow \mathrm{A}$ & 1347 & $15-\mathrm{gh}$ & PTT-EMD & $\begin{array}{l}266 ; 216937 \\
193859\end{array}$ \\
\hline 4411 del AG & 1465 & 15-gh/hh2 & PTT-EMD & 187805 \\
\hline 4439 del C & 1474 & 15-gh/hh2 & PTT-EMD & 177751 \\
\hline 4630 del GA & 1538 & 15-hh2 & PTT-EMD & 101206 \\
\hline
\end{tabular}

Numbering of nucleotides (nt) and codons is related to the GenBank file M74088 (nt 1 in position 1 of cDNA; codon 1 is first ATG at nucleotide 19 of cDNA). 
by PTT, was also submitted to EMD according to the presented protocol (see Subjects and methods). In total, 26 pathogenic APC mutations were identified in 75 FAP or AFAP patients (35\%). They represent twenty different DNA alterations (nine deletions, two insertions, eight nonsense mutations and one $\mathrm{GC} \rightarrow \mathrm{A}$ mutation) (Table 1). The mutations were located in exons $6,8,9,10,11$, and in fragments $a$ to $i$ of exon 15. Six patients carried the same $5 \mathrm{bp}$ deletion at codon 1309. This particular mutation represents nearly a quarter of all positive samples in this study. In total, six novel mutations were identified in this Belgian patient population in six different families. Table 2 describes the polymorphisms and variants identified by EMD. Clinical data for the patients harbouring new mutations are listed in Table 3.

In Figure 1 the cleavage profiles of three mutations located in amplicon c3-d of exon 15 are shown. The control sample, which is not cleaved by enzyme, gives a full-size peak of $790 \mathrm{bp}$. The heteroduplexes, present in the PCR products generated from heterozygous samples, are efficiently cleaved; two peaks can be observed for each sample. The normal and mutant homoduplexes are not cleaved and are detected as full-size peaks of lower intensity compared to the control sample.

The background level for EMD analysis of exon 6 was high, and interpretation of the results was difficult. Other conditions for hybridisation were applied $\left(95^{\circ} \mathrm{C}, 4 \mathrm{~min}\right.$; room temperature, $15 \mathrm{~min}$ ), but the background could not be decreased (results not shown). When PCR samples were loaded without having undergone an EMD reaction, the background was already present. This suggests that the PCR of this fragment caused the problems which we have not been able to solve. As an alternative, it was decided to screen this exon by DGGE, which enabled the detection of two nonsense mutations, R216X and R232X.

All the patients selected for this study have been screened for two variants located in exon 15 (fragment $\mathrm{g}-\mathrm{h})$ : I1307K and E1317Q. EMD has been performed in two successive steps: the PCR samples were studied alone, or mixed with a negative control prior to the EMD hybridisation step, which allows the detection of heterozygotes and homozygotes respectively. One patient (234429) was found to be a carrier of the $11307 \mathrm{~K}$ mutation; he was the only one detected in this series. Two patients (226582 and 180798) were heterozygotes for the E1317Q variant; no homozygote has been found in this series. The screening of a control population for the E1317Q allele detected one homozygous sample out of 180 controls (two alleles out of 360 or $0.6 \%$ ); in this same group, no heterozygous sample has been detected by EMD (details not shown).

\section{Discussion}

In this study we present the results of the EMD mutation scanning of the APC gene. The first part of the study compared EMD to the standard PTT method. Six deletions,

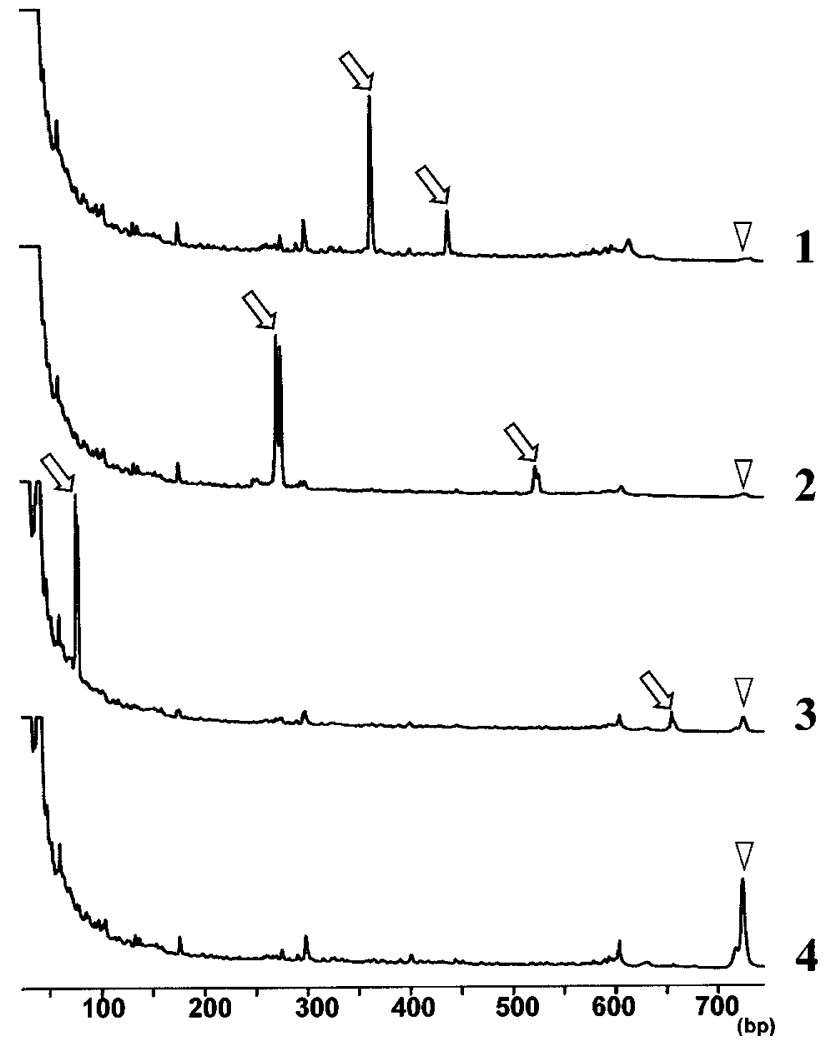

Figure 1 EMD analysis of APC exon 15 fragment c3-d. Cleavage profiles of three mutated samples and one control sample are shown. For PCR, forward and reverse primers labelled with FITC were used; the full-size product is $790 \mathrm{bp}$ and designated by a triangle. The comparison with a control patient (lane 4) makes it possible to pinpoint the specific peaks resulting from enzymatic cleavage of the two heteroduplexes present in each PCR product. Other fragments are present both in control and patients and result in background generated by the PCR itself and/or by the enzymatic reaction. Specific peaks are indicated by arrows. Lane 1: patient 38378 bearing Y935X, expected cleavage peaks of 357 and $433 \mathrm{bp}$; lane 2: patient 30913 bearing 2911 del AATA, expected peaks of 269 and 521 bp; lane 3: patient 175491 bearing 3104 del TT, expected peaks of 76 and $714 \mathrm{bp}$; lane 4: control patient with no specific cleavage products.

Table 2 Polymorphisms, unknown variants and missense mutations identified by EMD

\begin{tabular}{lccc}
\hline DNA change & Exons & Mutation & Comment \\
\hline TAC $\rightarrow$ TAT & 11 & Y486Y & polymorphism \\
GCA $\rightarrow$ GCG & 13 & A545A & polymorphism \\
TAC $\rightarrow$ TAT & 15 & Y935Y & polymorphism \\
ATA $\rightarrow$ ATT & 15 & $11055 \mathrm{I}$ & polymorphism \\
ATA $\rightarrow$ AAA & 15 & $11307 \mathrm{~K}$ & premutation \\
GAA $\rightarrow$ CAA & 15 & E1317Q & unknown variant \\
ACG $\rightarrow$ ACA & 15 & T1493T & polymorphism \\
GGA $\rightarrow$ GGG & 15 & G1678G & polymorphism \\
GAC $\rightarrow$ GTC & 15 & D1822V & polymorphism \\
CCA $\rightarrow$ CCG & 15 & P1960P & polymorphism \\
\hline
\end{tabular}


Table 3 Genotype-phenotype correlation of the novel mutations identified in this study

\begin{tabular}{|c|c|c|c|c|c|c|c|}
\hline \multirow[b]{2}{*}{ Mutation } & \multirow[b]{2}{*}{ Age of onset } & \multirow[b]{2}{*}{ Colon } & \multicolumn{3}{|c|}{ Number of adenomas } & \multirow[b]{2}{*}{ Osteomas } & \multirow[b]{2}{*}{ Phenotype } \\
\hline & & & Duodenum & Rectum & Fundic cystic glands & & \\
\hline 1494 del C & unknown & $>100$ & n.i. & n.i. & n.i. & n.i. & classical \\
\hline 2128 del G & 18 & $>100$ & yes & n.i. & no & no & classical \\
\hline 3104 del TT & 10 & 30 & yes & yes & yes & yes & classical \\
\hline 3614 ins $A$ & 23 & $>5000$ & no & n.i. & n.i. & n.i. & profuse \\
\hline $4057 \mathrm{GC}>\mathrm{A}$ & 13 & $>100$ & no & yes & n.i. & n.i. & classical \\
\hline 4439 del C & 27 & $>100$ & yes & no & yes & no & classical \\
\hline
\end{tabular}

n.i.: not investigated

one insertion, two substitutions, one $\mathrm{GC} \rightarrow \mathrm{A}$ mutation had been previously detected by PTT of exon 15 . For comparison with EMD, overlapping amplicons harbouring these 10 mutations were generated. Two cleavage fragments could be observed for all samples. The method has previously been applied in a hereditary non polyposis colorectal cancer (HNPCC) study: Otway and coworkers succeeded in the radioactive detection of $91 \%$ of all mutations in the $h M L H 1$ and $h M S H 2$ genes. They could not explain why six samples did not give any positive EMD pattern; two of these mutations were insertions. ${ }^{19}$

In the second part, we extended the EMD screening protocol. Based on the published mutation spectrum, it was decided to screen the entire coding region of the $A P C$ gene (see Subjects and methods).

With the analysis of 31 amplicons of the APC gene by EMD, only one unexplained complication was observed for analysis of exon 6 . The use of a primer, $74 \mathrm{bp}$ upstream of the exon, resulted in unspecific amplification. To circumvent the problem, this exon was screened by DGGE, for which the upstream primer was located close to the intron-exon boundary in comparison to the primer used for EMD.

Thus, the EMD method faces one intrinsic problem, ie the requirement to amplify relatively ample intronic sequences to warrant detection of variants within the coding region and flanking sequences: in this study, the PCR products were not purified before enzymatic digestion and gel analysis, with the consequence that the excess of labelled primers blurred the detection at the 5 ' end of the fragments. Small digested fragments (below $70 \mathrm{bp}$ ) are sometimes not detectable. This inconvenience can be avoided by the use of overlapping fragments (which is generally the case in the protocol presented here, and especially true for exon 15), or by the purification of PCR products prior to EMD. Alternatively, since both ends of the fragments are labelled, two cleavage peaks and thus reciprocal images are obtained, ie any abnormality will show up both at the proximal and distal end of the electropherogram.

Reported polymorphisms or variants of unknown significance are spread over the whole coding region, and they are also detected by EMD. For this study, cleavage peaks giving the same EMD profile with a frequency higher than 5\% in the FAP population were not further analysed by sequencing as they were considered as frequent polymorphisms in the Belgian population. A minor drawback is that these polymorphisms could interfere with the results as EMD would not distinguish between polymorphism and a pathogenic mutation located at about the same position because both profiles would be identical. This would be limited to point mutations that affect this specific nucleotide or the flanking nucleotides. The resolution of the method is such that differences of 1 or 2 bases are discernable. In the latter case, the fragment would thus be sequenced for confirmation.

Germline mutations in the exons 4, 9, and the 3' end of exon 15 have been associated with AFAP. ${ }^{7-9,20}$ In patient 202032 , with less than 10 polyps by the age of 62 years, a mutation was identified: an insertion at codon 357 in exon 9. This mutation has previously been described. ${ }^{20}$ The attenuated phenotype is explained by the position of the mutation in the alternatively spliced fragment of exon 9. In patient 179129, a truncating mutation (S457X) was detected in exon 10. This patient was diagnosed at the age of 41 years with only a few polyps $(<100)$ in colon and rectum. A sub-total colectomy has been performed, and since then clinical follow-up did not show any recurrence of adenomas (stomach normal). His phenotype can be classified as attenuated, which is rather unusual with regard to the position of the mutation. It had already been described in a FAP patient with a classical phenotype (at least 100 adenomas). ${ }^{21}$

Recent studies showed that apparently silent DNA changes can be responsible for a higher risk of colon cancer: the $A P C$ allele $\mathrm{I} 1307 \mathrm{~K}$ is known to increase the risk of transition from polyp to colorectal carcinoma in the Ashkenazi Jewish population. ${ }^{22,23}$ As a consequence of the ATA $\rightarrow$ AAA substitution, corresponding to I1307K, an (A) 8 tract is generated on the mutated allele. It has been demonstrated that this A stretch can cause polymerase slippage during replication and could act in this way as a 'premutation'. ${ }^{24}$ Patient 234429 who was heterozygote for this mutation is of Ashkenazi Jewish decent, had 10 polyps at diagnosis by the age of 58 years, and his phenotype is 
thus clearly attenuated. Our data is in agreement with the published results.

A second variant, E1317Q, has also been described as a more common $A P C$ variant that had been identified in $4 \%$ of AFAP patients with or without family history of colon cancer. ${ }^{12,25}$ In our series, two patients were heterozygous for the E1317Q allele. Patient 226582 had an attenuated phenotype (10 polyps by the age of 39 years), and no other alteration was identified after EMD analysis of $A P C$. The second $\mathrm{E} 1317 \mathrm{Q}$ carrier in this FAP population was patient 180798, who was also heterozygous for the S1194X nonsense (truncating) mutation in exon 15. Further genetic analysis of this family showed that his two children, both affected, have inherited both the nonsense and the missense mutations, while their mother is homozygous for the respective wild-type alleles. The missense mutation is thus localized on the allele harbouring the nonsense mutation. Hence, the significance of the E1317Q allele remains unclear. We did not identify heterozygotes in a series of 180 controls, but identified one control who was homozygous. The E1317Q has thus an allele frequency of $0.6 \%$ in this Belgian control population. The homozygous DNA sample was from a man of 86 years of age who had no clinical history of colon cancer. This suggests that this allele is probably not pathogenic. Our data is not in agreement with the inferences made by Lamlum et al., who identified two carriers in 503 population-based UK controls (allele frequency of $0.2 \%$ ), and seven in their series of 164 patients with multiple colorectal adenomas (3-96 tumors). ${ }^{25}$ Four out of the seven patients had a positive family history of colon cancer. The low number of adenomas may correspond to an attenuated form, which is similar to the phenotype observed in patient 226582. Definitely, a larger survey is required to settle this problem.

Based on genotype-phenotype correlations and mutation frequency in the APC gene that were published worldwide, the following screening protocol could be proposed:

(1) A partial analysis of exon 15: amplicons $\mathrm{g}-\mathrm{h}$ contains the frequent deletion at codon 1309 that is associated with classical (or more severe) FAP phenotype. The rare variants $\mathrm{I} 1307 \mathrm{~K}$ and $\mathrm{E} 1317 \mathrm{Q}$ will also be detected, but the phenotypic characteristics that could be associated with these two mutations have to be studied further.

(2) For classical FAP, the second frequent deletion at codon 1061 will be picked up by analysing amplicons $\mathrm{d}-\mathrm{e}$.

(3) Attenuated forms can be associated with mutations in exons 9 and 10. Mutations in exon 4 are also frequently associated with AFAP, although no positive sample has been found in this study.

(4) Rare frameshift mutations in the second part of exon 15 have been also associated with AFAP. Analysis of the region including the amplicons $\mathrm{k}-\mathrm{l}$ to $\mathrm{v}-\mathrm{W}$, is thus necessary to detect these truncating mutations.
In general, since the mutations are spread throughout the gene, one would have to complete a broader screening that also encompasses the first exons (no mutation has been found in the two first exons and in exon 10A), for routine diagnostics.

In total, 26 pathogenic mutations have been identified out of 75 FAP or AFAP patients after complete APC screening, and one more patient was heterozygous for the $11307 \mathrm{~K}$ variant. This corresponds to a rather low mutation detection rate. However, among the patients with a negative result after complete screening, there is a large portion of AFAP patients with evident familial history of polyposis. This group represents 22 AFAP out of the 48 patients who were negative upon screening. Recently, identical observations have been made by Friedl and coworkers: among 680 index patients that were screened for APC mutations, the mutation detection rate was weaker in AFAP patients than in patients diagnosed with more than 100 adenomas (31.7\% vs $58.2 \%$ respectively). ${ }^{26}$ There are different possibilities to explain the limited mutation detection rate in AFAP patients: (1) The methods we used (PTT, EMD) would not have detected large genomic deletions, as they both rely on PCR amplification of genomic DNA. PTT can detect large deletions if it is carried out on RNA. ${ }^{27}$ The use of polymorphic intragenic and extragenic markers (microsatellites or single nucleotide polymorphisms - SNP's) also allows the detection of loss of heterozygosity; ${ }^{28}$ (2) Possibly, mutations in other genes could also be the cause of the attenuated forms of hereditary colon cancer, like the HNPCC genes ( $h M L H 1$ and $h M S H 2)$, or in genes that are still unknown.

EMD is compatible with most laboratory equipment since one can choose between the use of radioactively labelled PCR products or the use of fluorescently labelled products for ALF DNA sequencers (Amersham Pharmacia Biotech) or ABI sequencers (Perkin Elmer). The latter allows the use of several dyes, which is more suitable for predicting the exact localisation of the mutation: forward and reverse primers can be labelled with different fluorophores. This also facilitates peak detection by making background signals from the two strands non-additive. We performed EMD detection on an ALF DNA sequencer, which allows the loading of 40 samples, and the use of one dye (fluorescein isothiocyanate or FITC). As both primers were labelled with the same dye, the detected peaks did not enable the prediction of exact localisation of the genetic alteration within the studied PCR product. The minor drawback of this setting is that direct sequencing of the two sites where the mutation is expected to be identified is then required.

For the Passport kit, the hands-on time is less important than for PTT, which proceeds in several incubation steps for signal enhancement and includes gel drying. The experimental time can be decreased by the use of sequencers that allow the loading of a large number of samples. High throughput can also be achieved by automation in pipet- 
ting and the use of capillary sequencers that avoid preparation and handling of sequencing gels.

In conclusion, we showed that the EMD technology presented here is rapid, easy, non-toxic and avoids the use of radioactive isotopes. The screening strategy comprises an EMD scanning of large overlapping PCR fragments covering the entire coding region of $A P C$. All mutations previously identified by PTT have been detected. In addition, the I1307K 'premutation' and nine polymorphisms (including the $\mathrm{E} 1317 \mathrm{Q}$ variant) have been identified. It is therefore an elegant alternative to PTT.

\section{Acknowledgements}

We thank M Smaers (Center for Human Genetics, UCL, Brussels) and $J$ Wijnen (Leiden University) for providing positive controls for APC mutations. We are grateful for advice in EMD mutation detection provided by Amersham Pharmacia Biotech. S Tejpar was supported by a grant of FWO - Flanders, Belgium.

\section{References}

1 Spirio L, Olschwang S, Groden J et al: Alleles of the APC gene: an attenuated form of familial polyposis. Cell 1993; 75: 951-957.

2 Groden J, Thliveris A, Samowitz W et al: Identification and characterization of the familial adenomatous polyposis coli gene. Cell 1991; 66: 589-600.

3 Nishisho I, Nakamura Y, Miyoshi Y et al: Mutations of chromosome 5q21 genes in FAP and colorectal cancer patients. Science 1991; 253: $665-669$.

4 Miyoshi Y, Nagase H, Ando H et al: Somatic mutations of the APC gene in colorectal tumors: mutation cluster region in the APC gene. Hum Mol Genet 1992; 1: 229-233.

5 Li C, Bapat B, Alman B: Adenomatous Polyposis Coli gene mutation alters proliferation through ist B-Catenin-regulatory function in aggressive fibromatosis (desmoid tumor). Am J Pathology 1998; 153: 709-714.

6 Nagase H, Miyoshi Y, Horii A et al: Correlation between the location of germ-line mutations in the APC gene and the number of colorectal polyps in familial adenomatous patients. Cancer Res 1992; 52: 4055-4057.

7 van der Luijt R, Meera Khan P, Vasen HFA et al: Germline mutations in the $3^{\prime}$ part of APC exon 15 do not result in truncated proteins and are associated with attenuated adenomatous polyposis coli. Hum Genet 1996; 98: 727 - 734 .

8 Bresinger JD, Laken SJ, Luce MC et al: Variable phenotype of familial adenomatous polyposis in pedigrees with $3^{\prime}$ mutation in the APC gene. Gut 1998; 43: 548-552.

9 Soravia C, Berk T, Madlensky L et al: Genotype-Phenotype correlations in attenuated adenomatous polyposis coli. Am J Hum Genet 1998; 62: 1290-1301.

10 Powell SM, Petersen GM, Krush AJ et al: Molecular diagnosis of familial adenomatous polyposis. N Engl J Med 1993; 329: $1982-$ 1987.
11 van der Luijt R, Meera Khan P, Vasen $\mathrm{H}$ et al: Rapid detection of translation-terminating mutations at the adenomatous polyposis coli (APC) gene by direct protein truncation test. Genomics 1994; 20: $1-4$.

12 Frayling I, Beck N, Ilyas M et al: The APC variants I1307K and E1317Q are associated with colorectal tumors, but not always with a family history. Proc Natl Acad Sci USA 1998; 95: $10722-$ 10727.

13 Pedemonte S, Sciallero S, Gismondi V et al: Novel germline APC variants in patients with multiple adenomas. Genes Chromos Cancer 1998; 22: 257-267.

14 Youil R, Kemper B, Cotton RGH: Detection of 81 of 81 known mouse B-globin promoter mutations with T4 endonuclease VIIthe EMC method. Genomics 1996; 32: 431-435.

15 Solaro P, Birkenkamp K, Pfeiffer P, Kemper B: Endonuclease VII of phage $\mathrm{T}_{4}$ triggers mismatch correction in vitro. J Mol Biol 1993; 230: $868-877$.

16 Del Tito BJ, Poff III HE, Novotny MA et al: Automated fluorescent analysis procedure for enzymatic mutation detection. Clin Chem 1998; 44: 731-739.

17 Sulekova Z, Ballhausen WG: A novel coding exon of the human adenomatous polyposis coli gene. Hum Genet 1995; 96: 469-471.

18 Olschwang S, Laurent-Puig P, Groden J, White R, Thomas G: Germline mutations in the first 14 exons of the adenomatous polyposis coli (APC) gene. Am J Hum Genet 1993; 52: 273-279.

19 Otway R, Tetlow N, Hornby J, Kohonen-Corish M: Evaluation of enzymatic mutation detection in hereditary nonpolyposis colorectal cancer. Hum Mut 2000; 16: 61-67.

20 van der Luijt R, Vasen HFA, Tops CMJ, Breukel C, Fodde R, Meera Khan P: APC mutation in the alternatively spliced region of exon 9 associated with late onset familial adenomatous polyposis. Hum Genet 1995; 96: $705-710$.

21 Wallis YL, Morton DG, McKeown CM, Macdonald P: Molecular analysis of the APC gene in 205 families: extended genotypephenotype correlations in FAP and evidence for the role of APC amino acid changes in colorectal cancer predisposition. J Med Genet 1999; 36: 14-20.

22 Stern HS, Viertelhausen S, Hunter AGW et al: APC I1307K increases risk of transition from polyp to colorectal carcinoma in Ashkenazi Jews. Gastroenterology 2001; 120: 392-400.

23 Gryfe R, Di Nicola N, Lal G, Gallinger S, Redston M: Inherited colorectal polyposis and cancer risk of the APC I1307K polymorphism. Am J Hum Genet 1999; 64: 378-384.

24 Laken SJ, Petersen GM, Gruber SB et al: Familial colorectal cancer in Ashkenazim due to a hypermutable tract in APC. Nature Genet 1993; 17: 79-83.

25 Lamlum H, Al Tassan N, Jaeger E et al: Germline APC variants in patients with multiple colorectal adenomas, with evidence for the particular importance of E1317Q. Hum Mol Genet 2000; 9: $2215-2221$.

26 Friedl W, Caspari R, Sengteller M et al: Can APC mutation analysis contribute to therapeutic decisions in familial adenomatous polyposis? Experience from 680 FAP families. Gut 2001; 48: $515-521$.

27 Su L-K, Steinbach G, Sawyer JC, Hindi M, Ward PA, Lynch PM: Genomic rearrangements of the APC tumor-suppressor gene in familial adenomatous polyposis. Hum Genet 2000; 106: 101-107.

28 De Rosa M, Scarano IM, Panariello L et al: Three submicroscopic deletions at the APC locus and their rapid detection by quantitative-PCR analysis. Eur J Hum Genet 1999; 7: 695-703. 